\title{
Percepções Técnicas e Sociais de Fatores que Impactam no Desempenho de Práticas de Estágio Supervisionado em Tempos de Pandemia
}

\author{
Emanuel Ferreira Coutinho ${ }^{1,2,3}$, Regis Pires Magalhães ${ }^{1,2,3}$, Virginia Farias de Sousa $a^{1,3}$ \\ ${ }^{1}$ NPI - Núcleo de Práticas em Informática \\ ${ }^{2}$ Programa de Pós-Graduação em Computação (PCOMP) \\ ${ }^{3}$ Universidade Federal do Ceará (UFC) - Quixadá - CE - Brasil \\ emanuel.coutinho@ufc.br, regismagalhaes@ufc.br, virginia.farias@ufc.br
}

\begin{abstract}
Changes in the routine of system development activities occur constantly due to several factors such as technologies, resources and exceptional situations. One of these activities is the supervised internship, which promotes contact with the professional reality, dialogues with more experienced professionals, and obtain exchange of experiences. Due to the COVID-19 pandemic, many supervised internship actions had to adapt to the reality of performing activities remotely. The objective of this work is to analyze students' perceptions of systems development practices during the pandemic period, in supervised internship activities. For this, a questionnaire was designed and a qualitative analysis with Grounded Theory. As major conclusions of the work, we obtained: the work environment is essential for a good performance of activities; an adequate infrastructure directly impacts productivity; and personal feelings and problems should be treated with care when performing remote activities.
\end{abstract}

Resumo. Mudanças na rotina de atividades de desenvolvimento de sistemas ocorrem constantemente devido a diversos fatores como tecnologias, recursos e situações excepcionais. Uma dessas atividades é o estágio supervisionado, que promove contato com a realidade profissional, dialogar com profissionais mais experientes, e obter troca de experiências. Devido à pandemia do COVID-19, muitas ações de estágio supervisionado tiveram que se adaptar a uma realidade de execução de atividades de forma remota. O objetivo deste trabalho é analisar percepções dos alunos nas práticas de desenvolvimento de sistemas durante o período da pandemia, em atividades de estágio supervisionado. Para isto, um questionário foi projetado e uma análise qualitativa com Grounded Theory. Como grandes conclusões do trabalho, obteve-se: o ambiente de trabalho é essencial para um bom desempenho das atividades; uma infraestrutura adequada impacta diretamente na produtividade; e sentimentos e problemas pessoais devem ser tratados com cuidado quando da execução de atividades remotas.

\section{Introdução}

Constantemente, pessoas e sociedade testemunham mudanças de conceitos, valores e tecnologias [Torcate et al. 2018]. Devido ao ciclo natural do homem em procurar descobrir e 
aprender novos objetos de estudo, viver em sociedade requer apropriar-se de conhecimentos e habilidades específicas dependendo de suas necessidades e do contexto em que está inserido. E em um cenário onde certas restrições são impostas devido a situações excepcionais no contexto nacional ou internacional, como é o caso da pandemia do COVID-19, adaptar atividades se torna um desafio ainda maior.

Em um curso de graduação, diversas experiências são proporcionadas aos alunos, sendo muitas delas de fundamentação teórica ou caráter prático. Umas das experiências práticas e que consta em diversos currículos de cursos são os estágios. Para profissionais ainda em formação, o estágio é um dos momentos mais importantes e significativos, sendo um momento que o futuro profissional tem a oportunidade de entrar em contato direto com a realidade profissional na qual será inserida, além de concretizar pressupostos teóricos adquiridos pela observação de determinadas práticas específicas e do diálogo com profissionais mais experientes, obtendo assim a troca de experiências [Torcate et al. 2018].

A conexão universidade-empresa tem crescido em importância ao longo dos anos [Ribeiro Mello et al. 2017], e o estágio supervisionado, normalmente realizado durante o curso de graduação, é obrigatório em várias carreiras, segundo a legislação brasileira, e é considerado uma das formas de cooperação entre universidade e empresa. Em diversas situações, o estágio é supervisionado por alguém com maior experiência na área. O objetivo de um estágio supervisionado é prover ao aluno uma experiência aplicada de assuntos relacionados ao seu curso, geralmente ocorrendo em empresas, para que a vivência seja mais plena, relacionado-se com outras pessoas, áreas de atuação e clientes, fora do ambiente de sala de aula. Diversos benefícios tanto para a academia quanto para a indústria são percebidos por experiências de um estágio supervisionado, tais como: interação com ambientes mais reais de empresas, mesmo que simulados; lidar com responsabilidades de entregas, prazos e pressão; e desenvolvimento de habilidades em equipe.

Porém, durante os anos de 2020 e 2021, especificamente devido à pandemia do COVID-19, muitas ações de estágio supervisionado tiveram que se adaptar a uma realidade de execução de atividades de forma remota. Com isso, muitos desafios surgiram, tais como: comunicação entre membros da equipe e clientes, infraestruturas inadequadas, acompanhamento das atividades e utilização de ferramentas.

Mesmo assim, apesar do período de pandemia, as empresas ainda enfrentam o desafio de aumentar a produtividade de sua equipe, a fim de reduzir custos de produção e permanecer competitivo na indústria de software [Aquino Junior e Meira 2009]. E para melhorar a produtividade do desenvolvedor, eles precisam selecionar e implementar práticas eficazes que são impactadas por diversos fatores humanos e organizacionais. Especificamente para o desenvolvimento de sistemas, que já possui ferramentas para tarefas remotas, como repositórios de artefatos, controle de versão e distribuição em tempo real, o impacto da pandemia também refletiu de diversas formas, tanto na tecnologia quanto no lado pessoal e social. Assim, torna-se interessante conhecer melhor os efeitos da pandemia sobre os alunos e quais os fatores que contribuem no desempenho das atividades.

Especificamente no campus Quixadá, da Universidade Federal do Ceará, há um programa de extensão, o Núcleo de Práticas em Informática (NPI), que visa o desenvolvimento de sistemas para a sociedade, e a vivência em um ambiente semelhante a uma empresa, no modelo de fábrica de software. Nesse ambiente, diversos alunos do campus 
realizam as atividades de estágio supervisionado, cumprindo obrigações curriculares e experiências de desenvolvimento de sistemas similares a ambientes reais.

E nesse cenário, o objetivo deste trabalho é analisar o discurso dos alunos que experimentaram práticas de desenvolvimento no NPI de sistemas durante o período da pandemia, do ponto de vista técnico e social, em atividades de estágio supervisionado. $\mathrm{O}$ restante deste trabalho está dividido nas seguintes seções: a Seção 2 apresenta uma breve contextualização do núcleo de práticas; a Seção 3 discute alguns trabalhos relacionados; a Seção 4 descreve a metodologia aplicada a este trabalho; a Seção 5 apresenta os resultados e análises; e por fim, na Seção 6 as conclusões e trabalhos futuros são apresentados.

\section{Núcleo de Práticas em Informática (NPI)}

O Núcleo de Práticas em Informática (NPI) foi criado com o objetivo de atender a comunidade acadêmica e a sociedade da região do Sertão Central do Ceará com soluções de Tecnologia da Informação e Comunicação (TIC). O NPI também atua com a participação dos alunos dos últimos semestres que precisam desenvolver atividades de estágio durante sua graduação. Com seu estabelecimento oficial em 2011, foram iniciados projetos com alunos concludentes do curso de Sistemas de Informação. Em 2013, alunos concludentes do curso de Engenharia de Software também começaram a atuar nos projetos. Em 2016, alunos de Ciência da Computação também começaram a atuar nos projetos. Em 2017, o primeiro aluno de Design Digital começou a estagiar no NPI. A integração dos alunos de vários cursos, atuando nos mesmos projetos, permite que os projetos explorem habilidades e competências específicas de cada curso.

O NPI é estruturado nos moldes de uma fábrica de software, com processo definido e projetos com clientes reais. Um processo de desenvolvimento foi elaborado e implantado como forma de padronizar as atividades dos alunos no desenvolvimento de software e incorporar melhores práticas de Engenharia de Software conforme metodologias, métodos e modelos de maturidade de processo que utilizados na indústria de software e academia. Os alunos participantes são orientados por uma equipe de professores orientadores e supervisionados por servidores técnico-administrativos. Também realizam registro de ponto, para o acompanhamento da frequência pelo coordenador de estágio. Atualmente o NPI é registrado como um programa de extensão com múltiplas perspectivas, já que atende necessidades da comunidade (fornecimento de soluções de TIC), dos alunos (provimento de estágio) e dos docentes (ampliação da experiência profissional). Além disso, ele também funciona como estágio curricular obrigatório para os alunos.

\section{Trabalhos Relacionados}

Algumas iniciativas relacionadas a estágio supervisionado foram descritas na literatura, descrevendo experiências desta atividade.

Ferreira et al. (2015) relataram um minicurso semipresencial de introdução à programação, vivenciado durante a disciplina de Estágio Supervisionado do curso de Licenciatura em Computação da Universidade de Pernambuco (Campus Garanhuns). A ideia foi evidenciar o ensino de conceitos básicos de Computação sob a perspectiva do ensino semipresencial, de forma a proporcionar um aprendizado divertido e motivador em um público da educação básica. Silva et al. (2015) apresentaram uma experiência em ensino de Computação no ensino fundamental, vivido em uma disciplina de Estágio 
Supervisionado do Curso de Licenciatura em Computação da Universidade de Pernambuco (Campus Garanhuns) enfatizando as experiências de um grupo de estudantes e professores no ensino de conceitos de Computação. Para a pesquisa, propôs-se o desenvolvimento de jogos para educandos do ensino fundamental, a elaboração de materiais didáticos pedagógicos, baseando-se na relação teoria-prática para a realização das atividades de ensino-aprendizagem, fundamentando ainda uma proposta de currículo para o ensino de Computação. Apesar de ambas pesquisas não serem voltadas para o desenvolvimento de aplicações, que é o escopo deste trabalho, ambas destacaram a importância do estágio supervisionado para alunos e profissionais.

Mendonça et al. (2020) apresentaram um relato de experiência na transição dos processos de desenvolvimento de um projeto de P\&D para trabalho remoto em casa, visando saber mais dos impactos da pandemia que exigiu repensar o escopo do aplicativo a ser desenvolvido e seu plano de lançamento, revisar os procedimentos de desenvolvimento, e mudar a forma de comunicação com as partes interessadas. Os resultados sugerem que: a transição para o trabalho em casa não afeta o desempenho da equipe de desenvolvimento, o uso de ferramentas de comunicação e desenvolvimento de software colaborativo (e.g. Slack, Discord e Bitbucket) são um substituição eficaz para práticas de desenvolvimento face a face, e a comunicação com as partes interessadas é o principal desafio ao ter que lidar com a interrupção das atividades presenciais. Assim como neste trabalho, buscou-se analisar as percepções e desafios dos colaboradores diante da pandemia e do trabalho remoto em casa, assim como aspectos de comunicação.

Oliveira et al. (2020) descreveram os resultados de um survey para entender os impactos do isolamento social devido à pandemia da COVID-19 na produtividade dos desenvolvedores de software brasileiros, focando na percepção dos desenvolvedores. Os resultados indicaram que a produtividade dos desenvolvedores aumentou, principalmente por haver menos interrupções ao longo do dia. A maioria dos respondentes afirmaram que gostariam de continuar trabalhando de forma remota. Em relação à proposta deste trabalho, também buscou-se entender aspectos da produtividade e percepções.

Bezerra et al. (2020) investigaram como fatores humanos e organizacionais podem influenciar a produtividade das equipes de software de empresas brasileiras em ambiente remoto durante a pandemia COVID-19, por meio de um survey e análise qualitativa. Os principais resultados foram: $74,1 \%$ dos participantes consideraram que têm produtividade boa ou excelente; interrupção externa, adaptação ao ambiente e problemas emocionais são os principais fatores que influenciam a produtividade; $93,1 \%$ consideram ter uma equipe colaborativa e $84,5 \%$ têm fácil comunicação com seus colegas de trabalho; 84,5 \% sentem-se motivados; e as principais sugestões de investimentos para as empresas são em: material ergonômico, infraestrutura de comunicação remota, assistência às práticas de saúde e redução do turnover. Aspectos sociais e de comunicação também são destacados em nossa pesquisa, além da análise das equipes.

A atividade de estágio supervisionado realizada durante o curso de graduação é obrigatória em várias carreiras, sendo oficializado pela legislação brasileira como uma das formas de cooperação entre universidade e empresa [Ribeiro Mello et al. 2017]. Ribeiro Mello et al. (2017) ao considerarem que essa interação é benéfica para ambos os lados, verificaram como alunos de uma instituição de ensino têm sido avaliados quanto aos aspectos comportamentais, conhecimento e comprometimento por parte dos conce- 
dentes. O objetivo foi conhecer os pontos fortes e fracos para alimentar o processo de modernização da educação. Notou-se que, em geral, os alunos foram bem avaliados, indicando o valor da formação obtida na instituição estudada, embora seja possível fazer diversas melhorias. Similar à proposta deste trabalho, também buscou-se analisar o comportamento dos alunos durante a experiência de estágio obrigatório, e também pontos fortes e fracos.

\section{Metodologia}

O objetivo principal da pesquisa é analisar o discurso dos alunos que experimentaram práticas de desenvolvimento de sistemas durante o período da pandemia, do ponto de vista técnico e social, em atividades de estágio supervisionado. Segundo Gil (2008), pesquisas descritivas objetivam a explanação das características de fenômenos em observação, ajudando a levantar opiniões. Além disso, algumas pesquisas descritivas acabam servindo para proporcionar uma nova visão do problema. Para a fase de levantamento e coleta dos dados, utilizou-se o instrumento de questionário. O público-alvo para a aplicação do questionários foram os alunos que estagiaram no NPI.

Este trabalho consistiu em duas etapas: aplicação de um questionário online e análise qualitativa dos resultados. Inicialmente um questionário online foi projetado para a obtenção de dados, apresentado na Tabela 1, composto por dois grupos de questões: questões demográficas, para identificar o perfil de quem responde à pesquisa; e questões de experiência sobre ferramentas, comunicação e dificuldades. As questões demográficas eram todas de múltipla escolha, e as questões de experiência possuíam questões de múltipla escolha (QE1, QE2 e QE3) e texto livre (QE4). O objetivo dessas questões foi conhecer as experiências e percepções dos alunos diante do uso de ferramentas para desenvolvimento e comunicação durante as atividades de estágio supervisionado em tempos de pandemia. Todos os alunos deram consentimento para a utilização das respostas. Os dados obtidos foram analisados de maneira quantitativa e qualitativa. Na análise quantitativa utilizou-se estatística descritiva, por meio de valores absolutos, porcentuais e gráficos, para representar e descrever os dados de caracterização dos participantes.

Tabela 1. Questões demográficas (QD) e de experiência (QE) do questionário

\begin{tabular}{|l|l|}
\hline QD1 & Qual seu curso de graduação? \\
\hline QD2 & Você permaneceu até o final do semestre estagiando no NPI em pelo menos um semestre? \\
\hline QD3 & Classifique o quanto sua infraestrutura é compatível / é adequada / é suficiente para as práticas. \\
\hline QE1 & Classifique seu nível de estresse relacionado às práticas do NPI durante a pandemia. \\
\hline QE2 & Na sua opinião, durante a pandemia, as ferramentas para desenvolvimento foram adequadas? \\
\hline QE3 & Na sua opinião, durante a pandemia, as ferramentas para comunicação foram adequadas? \\
\hline QE4 & Quais as maiores dificuldades encontradas para estagiar no NPI durante a pandemia? \\
\hline
\end{tabular}

A análise qualitativa deste trabalho foi inspirada nos procedimentos de análise apresentados em [Ferreira et al. 2018]. Nessa análise, procedimentos da metodologia Grounded Theory [Corbin e Strauss 2014] foram aplicados. A Grounded Theory visa criar uma teoria a partir dos dados coletados e analisados sistematicamente, sendo composta por três fases: (1) codificação aberta, (2) codificação axial e (3) codificação seletiva. Na codificação aberta, se executa uma quebra, análise, comparação, conceituação e categorização dos dados. Na codificação axial, associam-se as categorias às suas subcategorias, formando categorias mais relacionadas e densas. Por fim, na codificação seletiva, se identifica a categoria ou ideia central do estudo, correspondente à teoria na qual todas 
as categorias estão relacionadas. Strauss e Corbin explicam que o pesquisador pode utilizar apenas alguns passos para atingir seu objetivo de pesquisa [Corbin e Strauss 2014]. Então, nessa pesquisa, apenas as fases 1 e 2 da Grounded Theory foram utilizadas para a identificação das categorias e suas relações. Adicionalmente, para evitar tendências nas análises, outro pesquisador revisou o resultado. Esse processo de revisão consistiu de revisão das categorias e relações identificadas, discussão para alinhar discordâncias e refinar o resultado.

\section{Resultados e Análises}

A coleta dos dados ocorreu durante um período de três semanas em fevereiro de 2021. Todos os alunos do que estagiaram no NPI em 2020.1 e 2020.2 foram convidados a responderem o questionário (85 no total). Apenas 38 alunos responderam $(44,7 \%)$.

\subsection{Resultados}

As questões iniciais foram de caráter demográfico, para se conhecer o perfil.

QD1 indicou o curso de graduação do alunos, sendo que 13 são de Engenharia de Software (34,2\%), 12 de Ciência da Computação (31,6\%), 9 de Sistemas de Informação $(23,7 \%)$ e 4 de Design Digital (10,5\%). QD2 apontou se o aluno permaneceu até o final do semestre estagiando no NPI, contando com 31 sim $(81,6 \%)$ e 7 não $(18,4 \%)$. QD3 foi em relação à adequação da infraestrutura do aluno para a execução das atividades, exibindo com nível médio $16(42,1 \%)$, alto 9 (23,7\%), altíssimo $8(21,1 \%)$ e baixo 5 (13,2\%). Dos 7 que responderam não permaneceram até o final no NPI, 3 indicaram que a infraestrutura deles era altíssima e 1 alta, porém carece de mais investigação. Isso pode indicar que a infraestrutura é independente de onde o aluno trabalha, considerando trabalho remoto, desde que ela seja adequada para as atividades. A Figura 1 exibe essas três questões.

QE1 está relacionada ao nível de estresse do aluno relacionado às práticas do NPI durante a pandemia. O nível de estresse médio foi o maior apontado pelos alunos, com $18(47,4 \%)$, seguido de nível alto e baixo com a mesma quantidade de respostas (8 $21,1 \%$ ) e nível de estresse inexistente para 4 alunos (10,5\%). Em QE2, a maioria das respostas indicou que as ferramentas de desenvolvimento foram totalmente adequadas (21-55,3\%), seguido de parcialmente adequadas (15-39,5\%) e indiferente $(2-5,3 \%)$. Nenhuma resposta indicou que as ferramentas foram inadequadas. QE3 apresentou dados sobre a adequação das ferramentas de comunicação, com 26 respostas para totalmente adequadas $(68,4 \%), 11$ para parcialmente adequadas $(28,9 \%)$ e apenas 1 para indiferente $(2,6 \%)$. Nenhuma resposta para ferramentas de comunicação inadequadas. Dos 8 alunos que responderam nível de estresse alto, 7 indicaram que as ferramentas de comunicação
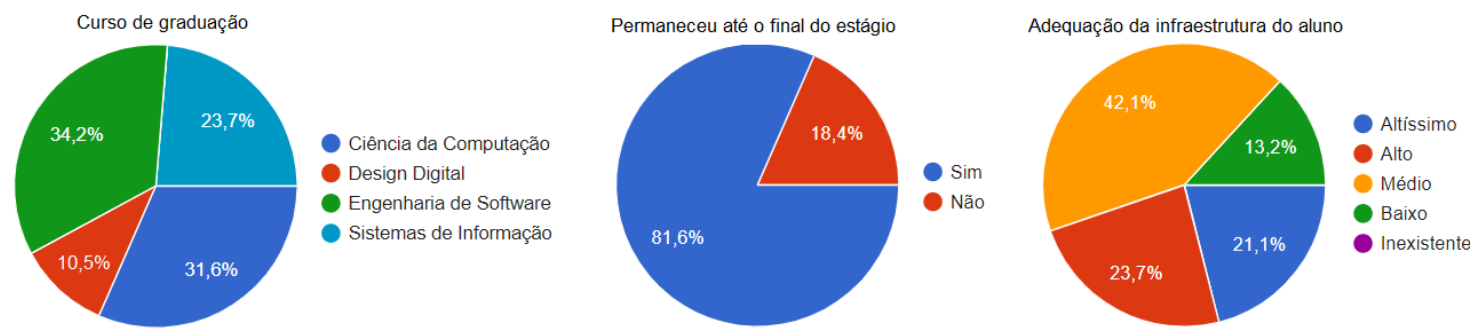

Figura 1. Questões demográficas (QD1, QD2 E QD3) 


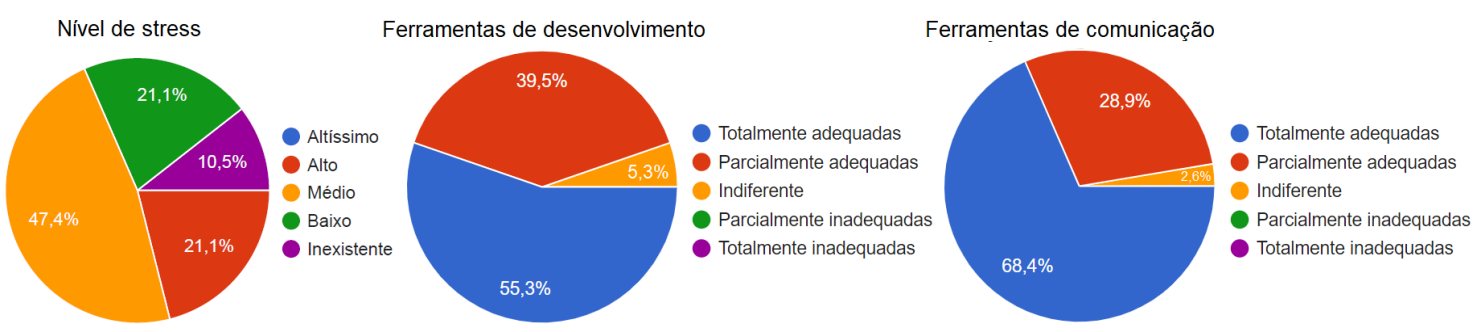

Figura 2. Questões de experiência (QE1, QE2 e QE3)

eram totalmente adequadas, e apenas 2 apontaram que as ferramentas de desenvolvimento eram totalmente adequadas. A Figura 2 exibe essas três questões. QE4 foi uma questão de texto livre, para indicar as dificuldades no período. Esta questão foi analisada com Grounded Theory, apresentada na subseção 5.2.

\subsection{Analise Qualitativa}

Considerou-se neste estudo verificar categorias relacionadas às dificuldades durante atividades de estágio supervisionado relatadas pelos alunos. Durante a análise qualitativa, 6 categorias foram identificadas, com a seguinte frequência de citações em ordem decrescente: Infraestrutura (24), Ambiente de Trabalho (13), Sentimentos (5), Pessoal (4). Comunicação (3) e Gestão (3), A Tabela 2 exibe a descrição de cada categoria. Quatro relacionamentos entre categorias foram identificados: Pessoal está associado com Ambiente de Trabalho, Ambiente de Trabalho está associado com Infraestrutura, Ambiente de Trabalho está associado com Sentimentos e Infraestrutura está associado com Gestão. Algumas citações dos participantes foram capturadas para destacar aspectos das questões de opinião, sendo que os participantes estão identificados de P1 a P38 para preservar a anonimidade. A Figura 3 exibe as categorias de dificuldades identificadas e seus relacionamentos.

Em relação à infraestrutura, a maioria das respostas foi em relação ao acesso à internet, como no discurso de $\mathbf{P 2}$ com "Normalmente problemas pessoais, como problemas na máquina ou falta de energia/internet". O ambiente de trabalho também foi muito mencionado como uma dificuldade, seja pela falta de local, seja por falta de concentração, como apontado por $\mathbf{P 1 9} \mathrm{em}$ "O ambiente dentro de casa não era muito propício". Em relação aos sentimentos, estes foram variados, e alguns relacionados ao estado de saúde, como comentado por $\mathbf{P 2 5}$ em "A dificuldade de resolver certos problemas, computador com programas em versões diferentes que causam problemas e a ansiedade devido a auto cobrança". O lado pessoal revelou fatores indiretos ao desenvolvimento, mas que impactaram no projeto, como na menção de $\mathbf{P 1 6}$ com "A internet, e os problemas pessoais relacionado a doenças com esse vírus". A comunicação teve algumas menções, como de

Tabela 2. Descrição das categorias identificadas na análise qualitativa

\begin{tabular}{|l|l|}
\hline Categoria & Descrição \\
\hline Ambiente de Trabalho & Local para execução das atividades do projeto (em casa, remoto, etc) \\
\hline Comunicação & Aspectos relacionados à comunicação na equipe (ferramentas, efeitos, etc) \\
\hline Gestão & Atividades de planejamento e acompanhamento do projeto \\
\hline Infraestrutura & Equipamentos, software, hardware, energia, conexão \\
\hline Pessoal & Problemas pessoais, aspectos individuais do aluno que impactam as atividades \\
\hline Sentimentos & Características e sentimentos que os alunos tiveram durante o período \\
\hline
\end{tabular}




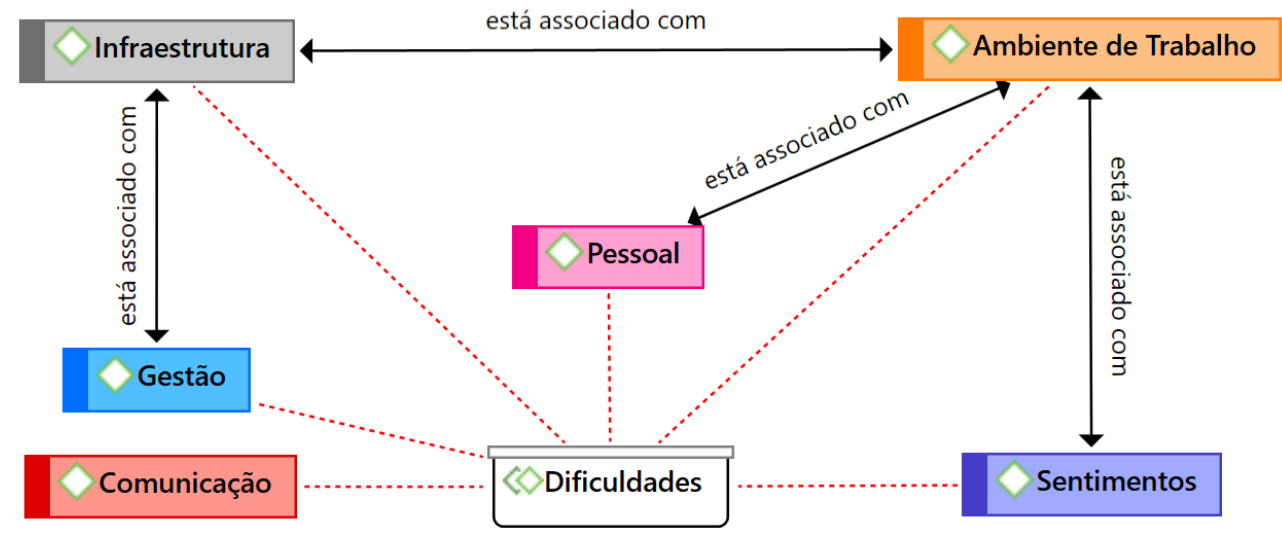

Figura 3. Categorias e relacionamentos das dificuldades em estagiar durante a pandemia

P27 com "Eventuais latências de comunicação devido a distância". A gestão descreveu mais situações de atividades do projeto, como em P36 com "Conciliar novos horários após voltar ao meu estado natal".

Quanto aos relacionamentos, "Pessoal está associado com Ambiente de Trabalho" foi destacado por P33 em "... Estar na casa dos meus pais, pois tive que conciliar estudo/estágio com ajudar meus pais e irmã com problemas familiares. É difícil se está em uma casa, eu que todos não tem o mesmo foco (como por exemplo em uma casa de alunos), as interrupções acabam prejudicando no desenvolvimento do estágio.". Para "Ambiente de Trabalho está associado com Infraestrutura", P9 fez o seguinte comentário: “... além do conhecimento das tecnologias de desenvolvimento, o ambiente as vezes pode não ser tão favorável comparado a forma presencial do estagio, a infraestrutura é completamente diferente do ambiente de trabalho utilizado na Universidade". Em relação a "Ambiente de Trabalho está associado com Sentimentos", $\mathbf{P 8}$ ressaltou "A adaptação ao home office pois exige bastante disciplina". E por fim, para "Infraestrutura está associado com Gestão", P10 destacou "Local adequado para realizar as atividades, máquina com capacidade baixas para realizar as atividades, stress da família as vezes atrapalhando a realização da atividade".

\subsection{Discussões}

As categorias infraestrutura e ambiente de trabalho se destacaram com mais menções nas respostas. A infraestrutura foi citada nos mais variados níveis (internet, equipamento, software, hardware, energia, etc). O campus possibilitava essas opções aos alunos, que passaram a ter que lidar com essas questões em suas residências. Além disso, o convívio social ajudava a resolver muitos desses problemas, pelo compartilhamento de recursos.

Quanto ao ambiente de trabalho, os problemas em casa, como doenças, não conseguir se dedicar e incômodos foram bastante citados. Um exemplo foi o comentário de $\mathbf{P 1 8}$ com "Falta de um ambiente, livre de barulhos e incômodos". No campus também há esses problemas, mas muitas vezes há a opção de mudança do local de trabalho (laboratórios, sala de aula, corredor, etc), e há também a inciativa de conscientização do corpo docente e técnico para que o ambiente seja mais adequado às atividades do projeto.

Filtrando a quantidade de respostas com nível de estresse alto, identificamos 8 res- 
postas. Cada resposta pode ter mais de uma categoria associada, e assim, discriminando por categoria, encontramos a seguinte quantidade de menções: 4 para infraestrutura, 3 para ambiente de trabalho, 2 pessoal, 2 sentimentos e 1 gestão. Isso indica que grande parte do estresse dos alunos está relacionado a possuir equipamentos e ambiente de trabalhos inadequados para as atividades, além de haver muitas interrupções.

O ambiente de trabalho foi a categoria que concentrou mais relacionamentos com outras categorias, sendo 3, com infraestrutura, pessoal e sentimentos. Com infraestrutura foi a maior quantidade de relações, como destacado por $\mathbf{P 1 0} \mathrm{com}$ "Local adequado para realizar as atividades, máquina com capacidade baixas para realizar as atividades, estresse da família as vezes atrapalhando a realização da atividade”. Com pessoal, problemas com família em casa foram citados, além de doenças, como no exemplo de P33 com “... Estar na casa dos meus pais, pois tive que conciliar estudo/estágio com ajudar meus pais e irmã com problemas familiares. É difícil se está em uma casa, eu que todos não tem o mesmo foco (como por exemplo em uma casa de alunos), as interrupções acabam prejudicando no desenvolvimento do estágio.". E com sentimentos, $\mathbf{P 2 5}$ mencionou "A dificuldade de resolver certos problemas, computador com programas em versões diferentes que causam problemas e a ansiedade devido a auto cobrança", sendo um exemplo de como a ansiedade prejudica o desempenho.

Diversos sentimentos foram relatados na pesquisa, como dificuldades na execução das atividades de estágio. Ansiedade, estresse com família e auto cobrança foram sentimentos citados pelos alunos. Além disso, problemas pessoais relacionados a doenças também colaboraram para dificuldades na execução das atividades.

As ferramentas de desenvolvimento foram em geral consideradas adequadas, com cerca de $95 \%$ de adequação. Isso foi um destaque porque há treinamentos iniciais na forma de auto estudo nas ferramentas de desenvolvimento, um estudo de caso, uma apresentação dos projetos desenvolvidos pelos alunos, e suporte técnico. Pode ser uma consequência dessa iniciativa antes do início pleno das atividades dos projetos. Exemplos de ferramentas de desenvolvimento utilizadas são os frameworks padronizados nos projetos, Gitlab e banco de dados, além das IDEs para programação em si.

Quanto às ferramentas de comunicação, cerca de 97\% das respostas apontaram com adequadas, o que de certa forma surpreendeu. A categoria de dificuldade comunicação obteve apenas 3 menções, o que estaria alinhado com essa alta adequação. Ferramentas de comunicação utilizadas foram o Slack para atividades mais do dia a dia do projeto, e o Google Meet para reuniões diárias. Mendonça et al (2020) comentaram que as ferramentas de comunicação e desenvolvimento de colaboração substituíram de maneira eficaz para práticas presenciais, sendo que a comunicação com as partes interessadas é o desafio a ser lidado. Em nossa pesquisa não houve menções a comunicação com clientes e fornecedores, e esse aspecto merece atenção. Bezerra et al. (2020) relataram que $84,5 \%$ dos respondentes possuem uma comunicação fácil na equipe. Isso de certa forma também ocorreu em nossa pesquisa, pois o porcentual de ferramentas adequadas foi muito alto. Porém, isso não necessariamente implica que a comunicação foi bem executada.

Por fim, o convívio social nas atividades de estágio de maneira presencial é algo que não pode ser dispensado, pois há diversas experiências sociais e técnicas que presencialmente teriam um melhor desempenho. Muitos dos sentimentos relatados podem ser 
minimizados remotamente, mas a realidade de cada um é uma situação a ser analisada.

\subsection{Limitações da Pesquisa}

Algumas limitações da pesquisa foram identificadas. A estrutura do questionário pode ter prejudicado um pouco a pesquisa, principalmente quanto ao teor subjetivo das questões. Mesmo com questões objetivas (de marcar), a forma na qual foram estruturadas pode ser entendida subjetivamente pelos alunos. Assim, essas questões pode ser mal interpretadas. Por exemplo: em relação a QD3, sobre a adequação da infraestrutura, o aluno poderia pensar que a infraestrutura é compatível, mas inadequada ou não suficiente para suas atividades. Uma sugestão para minimizar esse aspecto é ao se projetar um questionário, que sejam realizados pilotos com especialistas (análise do propósito, questões, opções, etc) antes de enviar aos respondentes. A quantidade de respostas também poderia ter sido maior, pois 38 alunos responderam (44,7\%), de 85 no total. Como a coleta dos dados ocorreu durante um período de três semanas, mesmo com uma situação de pandemia onde praticamente todos os alunos estavam estagiando de maneira remota, o ideal era ter mais respostas. Além disso, muitos alunos já não estagiavam mais no NPI, o que pode ter prejudicado o contato e resposta do questionário. Por fim, a qualidade das respostas de texto livre pode não ter sido tão boa. Diante dessas questões, é considerado que os dados não podem ser generalizados. Porém os resultados são de importância para o NPI e para identificar a percepção dos alunos que estagiaram nesse período de pandemia.

\section{Conclusão}

Este trabalho apresentou as percepções de alunos que desenvolveram atividades de estágio supervisionado em projetos de software. Especificamente durante o período, as atividades ocorreram de forma remota, explorando bastante o uso de tecnologias Esta pesquisa é parte de uma pesquisa mais ampla, que irá identificar diversos fatores que impactam no desenvolvimento de atividades de estágio supervisionado em tempos de pandemia e o impacto da utilização de tecnologias para o desenvolvimento e comunicação.

Como recomendações gerais para o NPI para melhorar o desempenho, sugerese primeiramente que a prática de avaliar as condições de trabalho dos alunos que estagiam, independente de pandemia e trabalho remoto, seja periodicamente conduzida. Como grandes conclusões do trabalho, tem-se: o ambiente de trabalho é essencial para um bom desempenho das atividades; uma infraestrutura minimamente adequada impacta diretamente na produtividade; sentimentos como estresse e problemas pessoais devem ser tratados com cuidado quando da execução de atividades remotas. Esse trabalho pode beneficiar os gestores do campus (diretores e coordenadores) auxiliando na tomada de decisão para uma melhoria das condições de estudo e trabalho, assim como os colaboradores do núcleo de práticas para melhor entender a situação dos alunos que realizam estágio supervisionado, e assim aprimorar processos. Como trabalhos futuros, pretende-se ampliar a pesquisa para os semestres subsequentes, considerando que ainda está sendo aplicada a modalidade de trabalho remota. Em sequência conduzir análises quantitativas e qualitativas com os dados obtidos, e propor melhorias para as práticas de estágio supervisionado durante o período em que as atividades estão ocorrendo de forma remota. Além disso, uma descrição de como os alunos estão se sentindo diante das dificuldades do momento auxiliaria os colaboradores do campus na melhoraria da qualidade do trabalho dos alunos e no bem estar geral. Por fim, para as dificuldades indicadas pelos alunos, investigar mais profundamente as causas e apontar possíveis soluções. 


\section{Referências}

Aquino Junior, G. e Meira, S. R. d. L. (2009). Towards effective productivity measurement in software projects. In 2009 Fourth International Conference on Software Engineering Advances, pages 241-249. IEEE.

Bezerra, C. I. M., de Souza Filho, J. C., Coutinho, E. F., Gama, A., Ferreira, A. L., de Andrade, G. L. a., e Feitosa, C. E. (2020). How human and organizational factors influence software teams productivity in covid-19 pandemic: A brazilian survey. In Proceedings of the 34th Brazilian Symposium on Software Engineering, SBES '20.

Corbin, J. e Strauss, A. (2014). Basics of Qualitative Research: Techniques and Procedures for Developing Grounded Theory. SAGE Publications, 4 edition.

Ferreira, M., de Lima, J., Santos, H., Oliveira, E., e do Nascimento, J. (2015). Computação para ensino médio na modalidade semipresencial: Uma experiência da disciplina de estágio supervisionado. In Anais do XXIII Workshop sobre Educação em Computação, pages 406-415.

Ferreira, T., Viana, D., Fernandes, J., e Santos, R. (2018). Identifying emerging topics and difficulties in software engineering education in brazil. In Proceedings of the XXXII Brazilian Symposium on Software Engineering, SBES '18.

Gil, A. C. (2008). Como elaborar projetos de pesquisa. Atlas, 4a. edition.

Mendonça, W., Costa, P. H. T., Cançado, E. C. R., Lima, F., Canedo, E. D., Bonifácio, R., e Amaral, L. H. V. (2020). From dusk till dawn: Reflections on the impact of covid-19 on the development practices of a ramp; d project. In Proceedings of the 34th Brazilian Symposium on Software Engineering, SBES '20.

Oliveira, E., Leal, G., Valente, M. T., Morandini, M., Prikladnicki, R., Pompermaier, L., Chanin, R., Caldeira, C., Machado, L., e de Souza, C. (2020). Surveying the impacts of covid-19 on the perceived productivity of brazilian software developers. In Proceedings of the 34th Brazilian Symposium on Software Engineering, SBES '20.

Ribeiro Mello, A. J., Villas Boas Mello, J. A., Quirino da Silva Souza, K., Lima de Souza, A. L., Alegre da Gama Afonso, H. C., Luiz da Cruz Barbosa de Souza, B., e Peixoto, A. (2017). University-enterprise cooperation for talent development: The case of supervised internship in a brazilian engineering university. In 2017 IEEE Global Engineering Education Conference (EDUCON), pages 969-976.

Silva, S., Barbosa, A., de Souza, A., da Silva, E., de Oliveira, M., da Silva Neto, S., e dos Santos, W. (2015). Relato de experiência de ensino de computação no ensino fundamental em estágio supervisionado da universidade de pernambuco no campus garanhuns. In Anais do XXIII Workshop sobre Educação em Computação.

Torcate, A. S., de Farias, M. U. F., da Silva, S. R. F., e Martins, C. S. (2018). A inserção de computação como disciplina no ensino fundamental: Desafios e conquistas em estágio supervisionado. In Anais do XXVI Workshop sobre Educação em Computação. 